\title{
ON PRODUCTIVE CLASSES OF FUNCTION RINGS
}

\author{
PAUL BANKSTON
}

\begin{abstract}
No nontrivial P-class ("P" for "productive") of rings of continuous real-valued functions can be category equivalent to any elementary $P$-class of finitary universal algebras.
\end{abstract}

0. Introduction. In this paper, "algebra" means "finitary universal algebra" in the sense of Birkhoff, and a class $\mathcal{K}$ of algebras will be viewed as a category by allowing all algebra homomorphisms as category morphisms. $K$ is productive or a $P$-class (resp. $S$-class) if th is closed under usual direct products (resp. subalgebras); ith is elementary if there is a set of first order axioms such that $t h$ is precisely the class of models of those axioms (see [5]).

We will be interested in category theoretic properties of classes of function rings. to wit: Let RCF denote the class of all rings of continuous real-valued functions $C(X)$ with topological spaces for domains. We ask which subclasses of RCF can be equivalent to "nice" classes of algebras (e.g. elementary $P$-classes, $S P$-classes, varieties, etc.).

0.1 Examples. (i) $\Re_{0}=\{C(X): X$ is zero-dimensional compact Hausdorff $\}$ is equivalent to the variety of Boolean algebras (see [6]).

(ii) $\mathscr{h}_{1}=\left\{C(X) \in \mathscr{h}_{0}: X\right.$ has no isolated points $\}$ is equivalent to the elementary $P$-class of atomless Boolean algebras.

There has come to be a growing list of negative results in this area. In [1] it is shown that $h=\{C(X): X$ is compact Hausdorff $\}$ cannot be equivalent to an $S P$-class; and in [3] it is shown also that $\mathcal{H}$ cannot be equivalent to a class $\mathcal{L}$ which is "representable" (i.e. free objects over singletons exist in $E$ ) and is either an elementary $P$-class or an $S$-class whose basic alphabet of operation symbols has cardinality less than that of the continuum. The major unsolved problem in this area is whether $T h$ is equivalent to any elementary $P$-class at all. In this paper we highlight the importance of the fact that products in $\mathcal{h}_{0}, \mathcal{K}_{1}$ and $\mathcal{K}_{\text {above are not the usual }}$ ones by proving results of which the following is an easy corollary.

0.2 TheOREM. No nontrivial P-subclass of RCF (i.e. one having more than the isomorphism type of the "degenerate" ring $0=C(\varnothing)$ ) can be category equivalent to an elementary P-class.

Received by the editors April 28, 1982 and, in revised form, August 9, 1982. 1980 Mathematics Subject Classification. Primary 08C05, 08C 10, 54C40.

Key words and phrases. P-classes, elementary classes, rings of continuous functions.

1983 American Mathematical Society $0002-9939 / 82 / 0000-0814 / \$ 02.00$ 
The proof employs the notion of "reduced product" in a category and will be presented in the next section.

1. Main results. We assume the reader to be familiar with the usual notion of reduced product in model theory [5]. The key observation, one which is well known, is that if $\mathscr{K}$ is any elementary $P$-class of finitary relational structures, then ultraproducts in $\mathcal{K}$ are simply direct limits of direct products (see $[2,3,4]$ for more details and references). This of course can be placed in a categorical context. We will show that most categorical reduced products in a $P$-subclass of RCF must be trivial (or nonexistent). This will immediately entail $(0.2)$ since the diagonal morphism from an algebra into an ultrapower in an elementary $P$-class is always a monomorphism.

Our notation regarding reduced products and powers comes from [5]: If $\left\langle A_{i}\right.$ : $i \in I\rangle$ is a family of relational structures of the same finitary type and $D$ is a filter on $I$ then $\prod_{D} A_{i}=\prod_{D}\left\langle A_{i}: i \in I\right\rangle$ is the reduced product with elements $a_{D}=\left\{a^{\prime} \in\right.$ $\left.\prod_{i \in I} A_{i}:\left\{i: a_{i}^{\prime}=a_{i}\right\} \in D\right\}$; if each $A_{i}$ is equal to $A$ then the reduced power is denoted $\Pi_{D} A$ and the natural diagonal embedding is denoted $d: A \rightarrow \Pi_{D} A$.

For $J \supseteq K \in D$, let $r_{J K}: \prod_{i \in J} A_{i} \rightarrow \prod_{i \in K} A_{i}$ be the natural restriction morphism. Then the associated direct $\operatorname{limit} \lim \left\langle\prod_{i \in J} A_{i}: J \in D\right\rangle$ is precisely the reduced product $\Pi_{D} A_{i}$ in the category of all relational structures. Moreover, if $\mathscr{K}$ is any elementary $P$-class then categorical ultraproducts in $\mathcal{K}$ are the usual ones. (N.B. It is possible to have an elementary class $\mathscr{K}$ which has unusual ultraproducts as a category (see [4]).)

Before we state our main results, we introduce the notion of "commuting system" of homomorphisms. Let $\left\langle X_{i}: i \in I\right\rangle$ be topological spaces, let $D$ be a filter on $I$, and let $X$ be any space. A "commuting system", in this context, is a family $\left\langle h_{J}: J \in D\right\rangle$ where, for $J \in D, h_{J}: \prod_{i \in J} C\left(X_{i}\right) \rightarrow C(X)$ is a homomorphism such that for all $J \supseteq K \in D, h_{K} \circ r_{J K}=h_{J}$. Our main concern is in the existence of certain commuting systems.

1.1 TheOREM. Assume $\left\langle X_{i}: i \in I\right\rangle$ is a family of topological spaces, $D$ is a free filter on $I$ (i.e. $\cap D=\varnothing), X$ is a space, there are no uncountable measurable cardinals at most $|I|(=$ the cardinality of $I)$, and $\left\langle h_{J}: J \in D\right\rangle$ is a commuting system. Then $X$ is empty.

Proof. It is well known (see [6]) that we lose no generality by assuming all of the above spaces to be realcompact Tichonov; and we can then invoke Theorem (10.6) of [6] to the effect that if $Y$ is realcompact and if $h: C(Y) \rightarrow C(X)$ is a ring homomorphism (N.B. $h(1)=1$. Hence $\operatorname{Hom}(0, C(X))={ }^{\circ} \varnothing$, unless $X=\varnothing$.) then there is a unique continuous $h^{\prime}: X \rightarrow Y$ such that, for all $f \in C(Y), h(f)=f \circ h^{\prime}$.

Let $\dot{U}_{i \in I} X_{i}$ denote the disjoint union of the spaces $X_{i}$; and let $p$ be a $z$-ultrafilter on $\dot{U}_{i \in I} X_{i}$ with the countable intersection property (c.i.p. = intersections of countable subfamilies of $p$ are nonempty). We show that $\dot{U}_{i \in I} X_{i}$ is realcompact by proving that $p$ must be fixed. Indeed let $g: \dot{U}_{i \in I} X_{i} \rightarrow I$ take $x$ to $i$ exactly when $x \in X_{i}$, and let $F=\left\{J \subseteq I: g^{-}[J] \in p\right\}$. One can check easily enough that $F$ is a countably complete ultrafilter on $I$ (e.g. $F$ is closed under superset since $g^{-}[J]=\dot{\cup}_{i \in J} X_{i}$ is always clopen, hence a zero set). Now there are no uncountable measurable cardinals 
at most $|I|$, hence $F$ must be fixed (= principal). Suppose $\{i\} \in F$. Then $X_{i} \in p$, and $p$ restricted to $X_{i}$ is a $z$-ultrafilter on $X_{i}$ with the c.i.p. Thus, since $X_{i}$ is realcompact, $p$ converges.

Now since $\prod_{i \in I} C\left(X_{i}\right)$ and $C\left(\dot{U}_{i \in I} X_{i}\right)$ are naturally isomorphic, we can consider each $h_{J}$ as a homomorphism from $C\left(\cup_{i \in J} X_{i}\right)$ to $C(X)$. Thus look at the "dual system" $h_{J}^{\prime}: X \rightarrow \dot{\cup}_{i \in J} X_{i}$. Letting $e_{J K}: \dot{\cup}_{i \in K} X_{i} \rightarrow \dot{\cup}_{i \in J} X_{i}$ be the natural embedding, $J \supseteq K \in D$ (an inclusion in this case), we note that the uniqueness of each $h_{J}^{\prime}$ ensures that all the appropriate diagrams commute (i.e. $e_{J K} \circ h_{K}^{\prime}=h_{J}^{\prime}$ for each $J \supseteq K \in D$ ). Since $\cap D=\varnothing$, this forces $X$ to be empty.

1.2 CoRollary. If $\mathcal{K}$ is a P-subclass of $R C F$ then reduced products in $\mathcal{K}$ are "trivial", in the sense that $\Pi_{D}^{\mathscr{K}} A_{i}$, the reduced product in $\mathcal{K}$, is zero whenever $D$ is a free filter on an index set whose cardinality is less than all uncountable measurable cardinals.

1.3 REMARK. The measurable cardinal hypothesis is necessary for 1.1 to work. For let $D$ be a free countably complete ultrafilter on a set $I$, and let each $X_{i}$ be a singleton. Then $\Pi_{D} C\left(X_{i}\right) \cong \mathbf{R}$ (= the ring of real numbers), by Corollary (4.2.8) in [5].

We can get the conclusion of 1.1 with altered hypotheses and more model theoretic techniques.

1.4 TheOREM. Assume $\left\langle X_{i}: i \in I\right\rangle$ is a family of spaces, $D$ is a countably incomplete ultrafilter on $I, X$ is a space, and $\left\langle h_{J}: J \in D\right\rangle$ is a commuting system. Then $X$ is empty.

Proof. Suppose, to the contrary, that there is a nonempty space $X$ for which a commuting system exists. If $\left\{i: X_{i}=\varnothing\right\}=J \in D$ then $h_{J}: \prod_{i \in J} C\left(X_{i}\right) \rightarrow C(X)$, being a ring homomorphism, forces $X$ to be empty. Since $D$ is an ultrafilter, we lose no generality by assuming that $X_{i} \neq \varnothing$ for each $i \in I$. For each $J \in D$ let $p_{J}$ : $\Pi_{i \in J} C\left(X_{i}\right) \rightarrow \Pi_{D} C\left(X_{i}\right)$ be the natural projection homomorphism. By properties of direct limits there is a unique homomorphism $h: \Pi_{D} C\left(X_{i}\right) \rightarrow C(X)$ such that for all $J \in D, h \circ p_{J}=h_{J}$. Now for each $i \in I$ let $d_{i}: \mathbf{R} \rightarrow C\left(X_{i}\right)$ be the diagonal embedding. Then the ultraproduct mapping $\Pi_{D} d_{i}: \Pi_{D} \mathbf{R} \rightarrow \Pi_{D} C\left(X_{i}\right)$ is a homomorphism. Now $D$ is an ultrafilter, hence $\Pi_{D} \mathbf{R}$ is a field by the Los Theorem. Therefore $h \circ \prod_{D} d_{i}$ is a homomorphism from a field into a nontrivial ring, hence an embedding. Let $d: \mathbf{R} \rightarrow \Pi_{D} \mathbf{R}, e: \mathbf{R} \rightarrow C(X)$ be the diagonal embeddings. It is a straightforward algebraic fact that there can be no other homomorphism $e^{\prime}: \mathbf{R} \rightarrow C(X)$, since $C(X)$ is a "diagonal" subring of a power of $\mathbf{R}$ (use the fact that the identity map is the only ring endomorphism on $\mathbf{R}$ ). Therefore $e=h \circ \Pi_{D} d_{i} \circ d$, and $\Pi_{D} \mathbf{R}$ embeds as a diagonal subring of $C(X)$. Since $D$ is countably incomplete, this ultrapower is $\omega_{1}$-saturated. We will obtain a contradiction once we prove the

LEMMA. No diagonal subring of a power of $\mathbf{R}$ is $\omega_{1}$-saturated.

Proof of Lemma. Let $A \subseteq \mathbf{R}^{I}$ be a diagonal subring which is $\omega_{1}$-saturated. For each $n \in \omega$, let $\phi_{n}(x)$ be the first order formula which says of $x$ that $x-n(=$ the 
result when the constantly $\boldsymbol{n}$ function is subtracted from the function $\boldsymbol{x}$ ) has a square root. $\phi_{n}(x)$ can be expressed in the first order language of rings with countably many additional constants. Clearly $\Phi(x)=\left\{\phi_{n}(x): n \in \omega\right\}$ is finitely satisfiable in $A$ : if $\Phi_{0}(x)=\left\{\phi_{n_{1}}(x), \ldots, \phi_{n_{k}}(x)\right\}$ then $A \vDash \phi_{n_{1}}\left[\max \left\{n_{1}, \ldots, n_{k}\right\}\right]$ for $i=1, \ldots, k$ since $\mathbf{R} \subseteq A$. So by $\omega_{1}$-saturicity, there is an $a \in A$ such that $a-n$ has a square root for each $n \in \omega$. That is, for each $i \in I$, the $i$ th coördinate $a_{i}$ of $a$ exceeds $n$ for all $n \in \omega$, a contradiction.

1.5 Corollary. If $\mathscr{K}$ is a P-subclass of RCF then ultraproducts in $\mathcal{K}$ are "trivial", in the sense that $\prod_{D}^{\mathfrak{K}} A_{i}$ is zero whenever $D$ is a countably incomplete ultrafilter.

\section{REFERENCES}

1. B. Banaschewski, On categories of algehras equivalent to a variety, Algebra Universalis (to appear).

2. P. Bankston, Reduced coproducts in the categon of compact Hausdorff spaces (to appear).

3. . Some obstacles to duality in topological algebra, Canad. J. Math. 34 (1982), 80-90.

4. P. Bankston and R. Fox, On categories of algebras equivalent to a quasivariety, Algebra Universalis (to appear).

5. C. C. Chang and H. J. Keisler, Model theory, North-Holland. Amsterdam, 1973.

6. L. Gillman and M. Jerison, Rings of continuous functions, Van Nostrand, Princeton, N. J., 1960.

Department of Mathematics, Statistics and Computer Science, Marquette University, MilwaukeE, Wisconsin 53233 